\title{
Avaliação de genótipos de cana-de-açúcar para início de safra na Microrregião Centro de Pernambuco
}

\author{
Pedro Henrique Neves de Souza ${ }^{1}$, Gerson Quirino Bastos ${ }^{2}$, Clodoaldo José da Anunciação Filho², \\ João de Andrade Dutra Filho ${ }^{3}$, Paulo Rocha Machado ${ }^{4}$
}

\section{RESUMO}

As diferentes respostas dos genótipos de cana-de-açúcar (Saccharum spp.), em relação à produtividade, quando submetidos a mudanças nos diferentes ciclos de colheita, representam problema para os agricultores e grande desafio para os melhoristas, sendo de interesse comum a identificação e obtenção de variedades que apresentem como características alta produtividade agroindustrial, ampla estabilidade, longevidade e excelente viabilidade econômica em sua exploração comercial. Objetivou-se, com este trabalho, avaliar o desempenho agroindustrial de 11 clones e 15 variedades de cana-de-açúcar, na microrregião canavieira da Zona Centro de Pernambuco, considerando-se os cultivos de cana planta, soca e ressoca, e eleger os genótipos mais produtivos. O experimento foi conduzido na área agrícola da Usina Petribú, município de Lagoa de Itaenga. Utilizou-se o delineamento experimental de blocos casualizados, com quatro repetições. Foram avaliadas as variáveis toneladas de pol por hectare (TPH), toneladas de cana por hectare (TCH), fibra (FIB), pol \% corrigida (PCC), pureza (PZA), teor de sólidos solúveis (BRIX) e açúcar total recuperável (ATR). Pela análise de variância, foram detectadas diferenças significativas entre os genótipos, indicando variabilidade genética. A alta estimativa da herdabilidade média para a variável TCH indicou elevada possibilidade de êxito na seleção, baseando-se neste importante componente de produção. Por meio da análise econômica, constata-se que as variedades RB92579 e RB93509 apresentam melhor desempenho para colheita no início da safra.

Palavras-chave: épocas de colheita, interação (genótipo x corte), parâmetros genéticos, Saccharum spp.

\section{ABSTRACT}

\section{Evaluation of sugarcane genotypes for initial season in the central microregion of Pernambuco}

Different responses of sugarcane (Saccharum spp.) genotypes, relative to yield, when submitted to several changes in harvest cycles represent a problem for farmers and a great challenge for breeders, being of common interest the identification and obtaining of varieties with high agroindustrial yield, wide stability, longevity and excellent economical viability on their commercial usage. This work aimed to evaluate the agroindustrial performance of 11 clones and 15 varieties of sugarcane in a cropland microregion in central zone of Pernambuco, considering the cultivation of cane plant, ratoon and re-ratoon, and to point out the most productive genotypes. The experiment was conducted in the agricultural area of Petribú mill, in the municipality of Lagoa de Itaenga. The experiment was arranged in a randomized block design with four replications. The variables evaluated were: tons of POL per hectare (TPH), tons of cane per

Recebido para publicação em 29/12/2011 e aprovado em 04/10/2012.

'Engenheiro-Agrônomo. Mestrando do Departamento de Agronomia, Universidade Federal Rural de Pernambuco, Rua Dom Manuel de Medeiros, s/n, Dois Irmãos, 52171-900, Recife, Pernambuco, Brasil. pedrohns@yahoo.com.br

${ }^{2}$ Engenheiro-Agrônomo, Doutor. Departamento de Agronomia, Universidade Federal Rural de Pernambuco, Rua Dom Manuel de Medeiros, s/n, Dois Irmãos, 52171-900, Recife, Pernambuco, Brasil. bastosgq@ hotmail.com; cjoseufrpe@hotmail.com

${ }^{3}$ Biólogo, Mestre. Doutorando do Departamento de Genética, Universidade Federal de Pernambuco, Avenida Professor Moraes Rego, 1235, Cidade Universitária, 50670-901, Recife, Pernambuco, Brasil. Pesquisador Cientifico, Estação Experimental de Cana-de-açúcar do Carpina da Universidade Federal Rural de Pernambuco, Rua Ângela Cristina Canto Pessoa Luna, s/n, Bairro Novo, 55810-000, Carpina, Pernambuco, Brasil. filho-dutra@ig.com.br (autor para correspondência).

${ }^{4}$ Engenheiro-Agrônomo. Estação Experimental de Cana-de-açúcar do Carpina da Universidade Federal Rural de Pernambuco, Rua Ângela Cristina Canto Pessoa Luna, s/n, Bairro Novo, 55810-000, Carpina, Pernambuco, Brasil. machadop_rocha@yahoo.com.br 
hectare (TCH), fiber (FIB), adjusted POL\% (PCC), purity (PZA), soluble solids content (BRIX) and total recoverable sugar (ATR). The analysis of variance detected significant differences among the genotypes indicating genetic variability. The high average herdability estimation for TCH indicated a high possibility of success in the selection based on such important yield component. Through the economical analysis it was verified that varieties RB92579 and RB93509 presented the best performance for the initial season harvest.

Key words: season times, genotype x harvest interaction, genetic parameters, Saccharum spp.

\section{INTRODUÇÃO}

O setor canavieiro possui um vasto portfólio de variedades, liberadas pelos programas de melhoramento existentes, ou obtidas por introdução varietal. Estes materiais apresentam características de interesse para a agroindústria canavieira, para atender as exigências de cada ambiente de produção, onde são submetidas à avaliação contínua, em experimentação, objetivando conhecer o seu comportamento, para que se possa proceder às recomendações de suas explorações, com base na viabilidade de seus aproveitamentos em cultivos comerciais (Melo et al., 2009).

A obtenção de novas variedades de cana-de-açúcar, que apresentem longevidade, isto é, elevada produtividade nos cultivos de cana planta, soca e ressoca, tem sido um dos principais objetivos dos programas de melhoramento genético, porque a degenerescência varietal tem ocasionado consideráveis perdas nos rendimentos agrícolas e industriais (Espironelo et al., 1988). Stupiello (2002) ressalta que algumas variedades chegam a apresentar um ciclo muito curto, com uma média de quatro a cinco cortes. Mamede et al., (2002) enfatizam que a base de sustentação do setor sucroenergético é o desenvolvimento contínuo de novas variedades, para substituição daquelas que apresentam o problema supracitado.

Nos programas de melhoramento da cana-de-açúcar, experimentos são conduzidos em diversas unidades produtoras, e colhidos, em sua maioria, em três épocas de colheita, com intuito de comparar o desempenho de novos materiais com aqueles explorados amplamente em cultivos comerciais (Ferreira et al., 2005).

No Estado de Pernambuco, experimentos com a cultura da cana-de-açúcar são colhidos em início de safra, que corresponde aos meses de setembro e outubro; meio de safra, a novembro e dezembro; e final de safra, a janeiro e fevereiro; em três ciclos de colheita, cana planta, cana soca e cana ressoca, respectivamente. Desta forma, de posse dos dados, tem-se a possibilidade de avaliar a magnitude das interações (genótipos x cortes / anos), que geralmente são significativas. Essas interações influenciam a seleção dos clones, estabelecendo maior probabilidade de se identificarem materiais que apresentem maior longevidade (Melo et al., 2009)
Melo et al., (2006), por meio de estudo envolvendo interação (cana-de-açúcar x ciclos de colheita), constataram que o comportamento das variedades não é estável, quando submetidas a diferentes ciclos de colheita. Estes autores concluíram que os melhoristas são obrigados a avaliar e a colher experimentos em várias safras agrícolas, para se estimar a interação e, mais ainda, procurar soluções para mitigar o seu efeito.

Objetivou-se, com este trabalho, avaliar o desempenho agroindustrial de 11 clones e 15 variedades comerciais de cana-de-açúcar, na microrregião canavieira da Zona Centro de Pernambuco, considerando-se os cultivos de cana planta, soca e ressoca, e eleger materiais mais produtivos.

\section{MATERIAL E MÉTODOS}

O experimento foi conduzido na microrregião Centro de Pernambuco (Koffler et al., 1986), em área agrícola da Usina Petribú, localizada no município de Lagoa de Itaenga ( $07^{\circ} 51^{\prime} 03$ ' S e $35^{\circ} 15^{\prime} 17^{\prime}$ ' $\left.\mathrm{W}\right)$, durante as safras agrícolas 2005/2006, 2006/2007 e 2007/2008. Foi utilizado o delineamento casualizado, em blocos completos, com quatro repetições, utilizando-se como tratamentos 26 genótipos de cana-de-açúcar, sendo 11 clones e 15 variedades comercialmente exploradas (Tabela 1). As parcelas experimentais foram compostas por cinco linhas de $8,0 \mathrm{~m}$ de comprimento, apresentando nas entrelinhas espaçamento de $1,0 \mathrm{~m}$, resultando numa área útil de 40 $\mathrm{m}^{2}$ por parcela.

Os dados foram coletados em três ciclos de colheita. O corte de cana planta foi realizado aos 15 meses de idade e, o corte de cana soca e ressoca, aos 12 meses, respectivamente. Foram avaliadas as seguintes variáveis: tonelada de cana por hectare (TCH), tonelada de pol por hectare (TPH), pol \% corrigida (percentual de sacarose no caldo da cana, PCC), pureza (PZA), fibra (FIB), teor de sólidos solúveis (BRIX) e açúcar total recuperável (ATR).

A produtividade por área (TCH) foi obtida por transformação do peso da parcela em Kg, por meio da seguinte equação (Peso total da parcela x 10 / área útil da parcela em $\mathrm{m}^{2}$ ). A variável tonelada de pol por hectare (TPH) foi calculada por meio da expressão (TCH x PCC / 100). Foi 
adotada a metodologia proposta por Fernandes (2003), para calcular as variáveis fibra (FIB), pol \% corrigida (PCC), pureza (PZA) e açúcar total recuperável (ATR). Com auxílio de refratômetro, foi mensurado o teor de sólidos solúveis (BRIX).

Para a análise individual de variância em cada ciclo de colheita, utilizou-se o modelo matemático aditivo linear $\mathrm{Y}_{\mathrm{ij}}=\mu+\mathrm{g}_{\mathrm{i}}+\mathrm{b}_{\mathrm{j}}+\varepsilon_{\mathrm{ij}}$, em que, $\mathrm{Y}_{\mathrm{ij}}$ é a observação do i-ésimo genótipo no j-ésimo bloco; $\mu$ : média geral; $\mathrm{g}_{\mathrm{i}}$ : é o efeito do i-ésimo genótipo; $b_{\mathrm{j}}$ : é o efeito do j-ésimo bloco; $\varepsilon_{\mathrm{ij} \text { : }}$ ó erro aleatório associado a cada observação $\mathrm{Y}_{\mathrm{ij}}$.

A análise de variância conjunta de experimentos foi realizada segundo o modelo estatístico apresentado por Cruz (2006): $\mathrm{Y}_{\mathrm{ijk}=} \mu+(\mathrm{b} / \mathrm{c})_{\mathrm{jk}}+\mathrm{g}_{\mathrm{i}}+\mathrm{c}_{\mathrm{k}}+\mathrm{gc}_{\mathrm{ijk}}+\varepsilon_{\mathrm{ijk}}$ em que: $\mathrm{Y}_{\mathrm{ijk}}$ : observação do i-ésimo genótipo, avaliado no j-ésimo bloco dentro do k-ésimo corte, $\mu$ : média geral do ensaio; (b/ c) ${ }_{\mathrm{jk}}$ : efeito do bloco k dentro do corte $\mathrm{j}$; $\mathrm{g}_{\mathrm{i}}$ : efeito do tratamento (ou genótipo) $\mathrm{i}$; $\mathrm{c}_{\mathrm{j}}$ : efeito do corte $\mathrm{k} ; \mathrm{gc}_{\mathrm{ik}}$ : efeito da interação entre o genótipo i e o corte $\mathrm{k} \mathrm{e} ; \varepsilon_{\mathrm{ijk}}$ : erro aleatório associado à observação ijk.

Foram determinados, como fixos, os efeitos de médias $(\mu)$ e genótipos (g), e aleatórios os efeitos do bloco (b),

Tabela 1. Identificação e procedência dos 11 clones e 15 variedades de cana-de-açúcar utilizadas em experimentos conduzidos na microrregião Centro de Pernambuco, Recife (PE), 2011

\begin{tabular}{lc}
\hline Genótipos & Procedência \\
\hline 1. RB867515 & RIDESA \\
2. RB92579 & RIDESA \\
3. SP81-3250 & COPERSUCAR \\
4. Q138 & AUSTRÁLIA \\
5. RB863129 & RIDESA \\
6. SP79-1011 & COPERSUCAR \\
7. RB93509 & RIDESA \\
8. RB75126 & RIDESA \\
9. RB942520* & RIDESA \\
10. SP78-4764 & COPERSUCAR \\
11. RB892700* & RIDESA \\
12. RB953180* & RIDESA \\
13. RB942898* & RIDESA \\
14. RB953281* & RIDESA \\
15. RB952900* & RIDESA \\
16. RB942991* & RIDESA \\
17. RB72454 & RIDESA \\
18. RB872552 & RIDESA \\
19. RB943365 & RIDESA \\
20. RB952675* & RIDESA \\
21. RB928064 & RIDESA \\
22. RB942849* & RIDESA \\
23. RB813804 & RIDESA \\
24. RB943161* & RIDESA \\
25. RB943066* & RIDESA \\
26. RB943538 & RIDESA \\
\hline
\end{tabular}

* Clones promissores do PMGCA/UFRPE/RIDESA corte (c), interação genótipo corte (gc) e o erro experimental $(\varepsilon)$. Foi aplicado o teste de Hartley para identificar homogeneidade de variâncias residuais e assim proceder à análise conjunta de experimentos. Os parâmetros genéticos foram estimados segundo Cruz (2006).

As médias foram agrupadas pelo teste de Scott-Knott (Scott \& Knott 1974), a 5\% de probabilidade. Baseandose na equação (Preço do quilo do ATR x ATR (kg/t) x $\mathrm{TCH}$ ), efetuou-se a análise de retorno econômico, para eleger os materiais mais produtivos. $\mathrm{O}$ processamento das análises estatísticas deu-se com o auxílio do aplicativo computacional genético-estatístico Genes (Cruz, 2006).

\section{RESULTADOS E DISCUSSÃO}

Os resultados das análises de variância individual em cada ciclo de colheita, para início de safra, estão apresentados na Tabela 2. Os resultados do teste F detectaram diferenças significativas a $1 \%$ de probabilidade $(\mathrm{P}<0,01)$ entre os genótipos, para todas as variáveis estudadas, nos três ciclos de colheita, exceto para a variável fibra (FIB), mostrando-se não significativa apenas no primeiro corte. Este resultado evidencia a existência de alto grau de variabilidade genética, entre os genótipos estudados, para todos esses caracteres que, de acordo com Bastos et al., (2003), estão entre os mais importantes componentes de produção em cana-de-açúcar.

Os valores do coeficiente de variação experimental (CV\%), de acordo com a classificação proposta por Gomes (1990), foram considerados baixos, para as variáveis PCC, FIB, PZA, BRIX e ATR, e médios, para as variáveis TCH e TPH, indicando boa precisão experimental. Em relação às variáveis TCH, TPH, PCC e ATR, observa-se maior variância genética para os três ciclos de colheita considerados, sendo superior a variância ambiental, indicando, de acordo com Dutra Filho et al., (2011a), que a expressão desses caracteres nos ciclos de colheita considerados é devida, sobretudo, a efeitos genéticos, sendo eficaz na identificação de indivíduos superiores para serem explorados nos locais onde se pratica a seleção (Tabela 2). Dutra Filho et al., (2011b), avaliando progênies de cana-de-açúcar na fase $\mathrm{T} 1$, encontrou resultados semelhantes para as variáveis TCH e TPH.

Ainda na Tabela 2, observa-se, com relação às variáveis TCH, TPH, PCC e ATR, que as estimativas da herdabilidade média $\left(h_{\mathrm{m}}^{2}\right)$, apresentaram valores que oscilaram de média a alta magnitude, nos três ciclos de colheita. Sendo a herdabilidade a proporção herdável da variabilidade total, este resultado é indicativo de sucesso na sua recombinação e no processo de seleção (Gonçalves et al., 2007).

Em relação aos coeficientes de variação genética (Tabela 2), observa-se que, para os caracteres TPH e TCH, 
nos três ciclos de colheita, foram obtidos valores acima de dez. De acordo com Oliveira et al., (2008), quando os coeficientes de variação genética atingem valores acima de dez é sinal da ocorrência de alto grau de variabilidade genética, indicando que a maior porção da variabilidade genética e os maiores ganhos na seleção seriam obtidos para esses caracteres.

Encontram-se na Tabela 3 os resultados dos sete caracteres agroindustriais, submetidos à análise de variância conjunta, avaliada em grupos de experimentos e conduzidos na fase de competição de variedades, para a $1^{a}$ época de colheita (início de safra).

Com base nos resultados do teste F, identificam-se diferenças significativas a $1 \%$ de probabilidade $(\mathrm{P}<0,01)$, entre os genótipos, para todos os caracteres em apreço. Esse resultado reflete, como nas análises individuais, a ocorrência de variabilidade genética entre os materiais genéticos considerados, quando avaliados em diferentes

Tabela 2. Resumo da análise individual de variância dos genótipos de cana-de-açúcar em cada corte cana planta, cana soca e ressoca, Lagoa de Itaenga-PE, anos agrícolas 2005/2006, 2006/2007 e 2007/2008, Recife (PE), 2011

\begin{tabular}{|c|c|c|c|c|c|c|c|c|c|c|}
\hline \multirow{2}{*}{ Cortes } & \multirow{2}{*}{ Variáveis } & \multicolumn{2}{|c|}{ Quadrado Médio } & \multirow{2}{*}{$\begin{array}{c}\text { Média } \\
\text { geral }\end{array}$} & \multirow{2}{*}{ CV $(\%)$} & \multirow{2}{*}{$\mathrm{CVg}$} & \multirow{2}{*}{$\hat{\sigma}_{\mathrm{g}}^{2}$} & \multirow{2}{*}{$\hat{\sigma}_{a}^{2}$} & \multirow{2}{*}{$\mathbf{h}^{2}{ }_{\mathrm{m}} \%$} & \multirow{2}{*}{$\mathrm{CVg} / \mathrm{CVe}$} \\
\hline & & $\overline{\text { Genótipos }}$ & Resíduo & & & & & & & \\
\hline \multirow{7}{*}{$1^{\circ}$ corte } & $\mathrm{TCH}$ & $386.96 * *$ & 87.74 & 73.27 & 12.78 & 11.80 & 74.80 & 21.93 & 77.32 & 0.92 \\
\hline & ТРH & $6.67 * *$ & 1.80 & 9.57 & 14.04 & 11.51 & 1.21 & 0.45 & 72.89 & 0.82 \\
\hline & PCC & $3.88 * *$ & 0.71 & 13.21 & 6.38 & 6.75 & 0.79 & 0.17 & 81.71 & 1.05 \\
\hline & FIB & $1.01 \mathrm{~ns}$ & 0.72 & 12.9 & 6.61 & 2.08 & 0.07 & 0.18 & 0.07 & 0.31 \\
\hline & PZA & $32.00 * *$ & 5.05 & 83.36 & 2.69 & 3.11 & 6.73 & 1.26 & 84.20 & 1.15 \\
\hline & BRIX & $3.68 * *$ & 0.60 & 18.97 & 4.09 & 4.62 & 0.77 & 0.15 & 83.62 & 1.13 \\
\hline & ATR & $423.27 * *$ & 82.61 & 133.8 & 6.79 & 6.89 & 85.16 & 20.65 & 80.48 & 1.01 \\
\hline \multirow{7}{*}{$2^{\circ}$ corte } & $\mathrm{TCH}$ & $506.84 * *$ & 123.35 & 84.39 & 13.15 & 11.60 & 95.87 & 30.83 & 75.66 & 0,90 \\
\hline & TPH & $8.50 * *$ & 2.55 & 11.99 & 13.31 & 10.17 & 1.48 & 0.63 & 69.99 & 0.76 \\
\hline & PCC & $3.04 * *$ & 1.04 & 14.27 & 7.17 & 4.94 & 0.49 & 0.26 & 65.56 & 0.68 \\
\hline & FIB & $1.00 * *$ & 0.34 & 13.24 & 4.42 & 3.06 & 0.16 & 0.08 & 65.75 & 0.69 \\
\hline & PZA & $15.79 * *$ & 7.21 & 84.88 & 3.16 & 1.72 & 2.14 & 1.80 & 54.29 & 0.54 \\
\hline & BRIX & $4.17 * *$ & 1.37 & 20.28 & 5.78 & 4.12 & 0.70 & 0.34 & 67.07 & 0.71 \\
\hline & ATR & $201.64 * *$ & 72.90 & 141.40 & 6.03 & 4.01 & 32.18 & 18.22 & 63.84 & 0.66 \\
\hline \multirow{7}{*}{$3^{\circ}$ corte } & $\mathrm{TCH}$ & $697.78 * *$ & 139.82 & 66.38 & 17.81 & 17.79 & 139.49 & 34.95 & 79.96 & 1,0 \\
\hline & TPH & $10.73 * *$ & 2.55 & 8.72 & 18.34 & 16.39 & 2.04 & 0.63 & 76.16 & 0,90 \\
\hline & PCC & $2.65 * *$ & 0.70 & 13.26 & 6.31 & 5.27 & 0.48 & 0.17 & 73.62 & 0.83 \\
\hline & FIB & $2.17 * *$ & 0.49 & 13.74 & 5.14 & 4.70 & 0.41 & 0.12 & 76.99 & 0.91 \\
\hline & PZA & $21.14 * *$ & 6.44 & 83.58 & 3.03 & 2.29 & 3.67 & 1.61 & 69.50 & 0.75 \\
\hline & BRIX & $3.39 * *$ & 0.87 & 19.31 & 4.84 & 4.10 & 0.62 & 0.21 & 74.14 & 0.84 \\
\hline & ATR & $160.55^{* *}$ & 44.13 & 132.87 & 4.99 & 4.06 & 29.10 & 11.03 & 72.51 & 0.81 \\
\hline
\end{tabular}

**, * Significativo a 1 e $5 \%$ de probabilidade respectivamente pelo teste $\mathrm{F}$

${ }^{(n s)}$, não significativo, coeficiente de variação $(\mathrm{CV} \%)$, coeficiente de variação genética $(\mathrm{CVg})$, variâncias genética $\left(\hat{\sigma}_{g}^{2}\right)$ e ambiental $\left(\hat{\sigma}_{a}^{2}\right)$, herdabilidade média $\left(\mathrm{h}^{2} \mathrm{~m} \%\right)$ e índice b $(\mathrm{CVg} / \mathrm{CVe})$ para $1^{\mathrm{a}}$ época de corte (início de safra)

Tabela 3. Resumo da análise de variância conjunta, avaliada em grupos de experimentos conduzidos na fase de competição de variedades na $1^{\mathrm{a}}$ época de colheita (início de safra), na microrregião Centro de Pernambuco, Usina Petribú, Lagoa de Itaenga-PE, anos agrícolas 2005/2006, 2006/2007 e 2007/2008, Recife (PE), 2011

\begin{tabular}{|c|c|c|c|c|c|c|c|c|}
\hline \multirow{2}{*}{ F.V. } & \multirow{2}{*}{ G.L. } & \multicolumn{7}{|c|}{ Quadrados médios } \\
\hline & & TPH & TCH & FIB & PCC & PZA & BRIX & ATR \\
\hline Genótipos & 25 & $13.83 * *$ & $1170.51 * *$ & $2.65 * *$ & $5.73 * *$ & $40.64 * *$ & $6.88 * *$ & $469.08 * *$ \\
\hline Cortes & 2 & $300.51 * *$ & $8594.35 * *$ & $18.44 * *$ & $37.51 * *$ & $69.89 *$ & $47.88 * *$ & $2274.18 * *$ \\
\hline $\mathrm{G} \times \mathrm{C}$ & 50 & $6.04 * *$ & $210.53 * *$ & $0.76^{*}$ & $1.93 * *$ & $14.14 * *$ & $2.18 * *$ & $158.19 * *$ \\
\hline Resíduo & 225 & 2.30 & 116.97 & 0.52 & 0.82 & 6.23 & 0.95 & 66.55 \\
\hline Médias & & 10.09 & 74.68 & 13.29 & 13.58 & 83.94 & 19.52 & 136.03 \\
\hline C.V (\%) & & 15.04 & 14.48 & 5.44 & 6.66 & 2.97 & 4.99 & 5.99 \\
\hline$>$ QMR / <QMR & & 1.41 & 1.59 & 2.11 & 1.49 & 1.42 & 2.27 & 1.87 \\
\hline
\end{tabular}

** e * Significativo a 1 e $5 \%$ de probabilidade respectivamente, pelo teste $\mathrm{F}$

(ns), não significativo, pelo teste $\mathrm{F}$

(G x C), Interação genótipo x Corte

Rev. Ceres, Viçosa, v. 59, n.5, p. 677-683, set/out, 2012 
anos agrícolas, indicando uma situação favorável para o melhoramento. Melo et al., (2006) apresentaram resultados concordantes com os deste trabalho, confirmando grande potencial dos clones e variedades para seleção e fins de melhoramento.
Em relação aos ciclos de colheita considerados (cana planta, soca e ressoca), foram identificadas diferenças significativas a $1 \%$ de probabilidade $(\mathrm{P}<0,01)$, para todas as variáveis, TPH, TCH, FIB, PCC, BRIX e ATR, e, a $5 \%$ de probabilidade $(\mathrm{P}<0,05)$, para a variável PZA. Constata-se

Tabela 4. Parâmetros estimados em três cortes das características avaliadas em fase de competição de variedades na $1^{\mathrm{a}}$ época de colheita (início de safra) em experimentos, conduzidos na microrregião Centro de Pernambuco, Usina Petribú, Lagoa de Itaenga-PE, anos agrícolas 2005/2006, 2006/2007 e 2007/2008, Recife (PE), 2011

\begin{tabular}{lrrrrr}
\hline \multirow{2}{*}{ Variáveis } & \multicolumn{5}{c}{ Parâmetros Genéticos } \\
\cline { 2 - 6 } & $\varphi_{\mathrm{g}}^{2}$ & \multicolumn{1}{c}{$\hat{\sigma}_{\mathrm{gc}}^{2}$} & $\mathbf{h}^{\mathbf{2}} \mathbf{C}$ & $\mathbf{C V}_{\mathrm{g}}$ & $\mathbf{C V g} / \mathbf{C V e}$ \\
\hline TPH & 0.64 & 0.89 & 56.32 & 7.98 & 0.53 \\
TCH & 79.99 & 22.49 & 82.01 & 11.97 & 0.82 \\
FIB & 0.15 & 0.05 & 71.01 & 2.98 & 0.54 \\
PCC & 0.31 & 0.26 & 66.25 & 4.14 & 0.62 \\
PZA & 2.20 & 1.90 & 65.19 & 1.77 & 0.59 \\
BRIX & 0.39 & 0.29 & 68.21 & 3.20 & 0.64 \\
ATR & 25.90 & 22.02 & 66.27 & 3.74 & 0.62 \\
\hline
\end{tabular}

$\varphi_{\mathrm{g}}^{2}$ : Componente de variância genética; $\hat{\sigma}_{\mathrm{gc}}^{2}$ : Componente de variância da interação genótipo corte; $\mathrm{h}^{2} \%$ : Herdabilidade média; $\mathrm{CV}_{\mathrm{g}}$ : Coeficiente de variação genética; $\mathrm{CVg} / \mathrm{CVe}$ : Índice b

Tabela 5. Valores médios dos TPH, TCH, FIB, PCC, PZA e ATR avaliados na fase de competição de variedades na $1^{\mathrm{a}}$ época de colheita (início de safra) considerando os cultivos de cana planta, soca e ressoca (três cortes) em grupos de experimentos, conduzidos na microrregião Centro de Pernambuco, Usina Petribú, Lagoa de Itaenga-PE, anos agrícolas 2005/2006, 2006/2007 e 2007/2008, Recife (PE), 2011

\begin{tabular}{|c|c|c|c|c|c|c|c|}
\hline Genótipos & TPH & TCH & FIB ( \%) & $\operatorname{PCC}(\%)$ & $\operatorname{PZA}(\%)$ & $\operatorname{BRIX}(\%)$ & $\operatorname{ATR}(\mathrm{kg} / \mathrm{t})$ \\
\hline Q138 & $9.13 \mathrm{~b}$ & $69.79 c$ & $13.12 \mathrm{~b}$ & $13.12 \mathrm{~b}$ & $83.28 \mathrm{a}$ & $18.95 \mathrm{~b}$ & $131.79 \mathrm{~b}$ \\
\hline RB72454 & $10.55 \mathrm{a}$ & $76.79 \mathrm{c}$ & $12.95 \mathrm{~b}$ & $13.68 \mathrm{a}$ & $85.20 \mathrm{a}$ & $19.27 \mathrm{~b}$ & $135.77 \mathrm{a}$ \\
\hline RB75126 & $10.65 \mathrm{a}$ & $82.10 \mathrm{~b}$ & $12.12 \mathrm{~b}$ & $13.03 \mathrm{~b}$ & $83.02 \mathrm{a}$ & $18.56 \mathrm{~b}$ & $130.69 \mathrm{~b}$ \\
\hline RB813804 & 8.94 b & $62.0 \mathrm{c}$ & $13.46 \mathrm{a}$ & $14.48 \mathrm{a}$ & $84.64 \mathrm{a}$ & $20.75 \mathrm{a}$ & $144.91 \mathrm{a}$ \\
\hline RB863129 & $10.38 \mathrm{~b}$ & $78.18 \mathrm{c}$ & $12.71 \mathrm{~b}$ & $13.20 \mathrm{~b}$ & 80.96 b & $19.44 \mathrm{~b}$ & $133.07 \mathrm{~b}$ \\
\hline RB867515 & $11.93 \mathrm{a}$ & $84.64 \mathrm{~b}$ & $13.26 \mathrm{a}$ & $14.03 \mathrm{a}$ & $85.15 \mathrm{a}$ & $19.85 \mathrm{a}$ & $138.73 \mathrm{a}$ \\
\hline RB872552 & $9.48 \mathrm{~b}$ & $66.41 \mathrm{c}$ & $13.68 \mathrm{a}$ & $14.10 \mathrm{a}$ & $84.76 \mathrm{a}$ & $20.21 \mathrm{a}$ & $140.27 \mathrm{a}$ \\
\hline RB892700* & $10.23 \mathrm{~b}$ & $72.06 \mathrm{c}$ & $13.77 \mathrm{a}$ & $14.15 \mathrm{a}$ & $85.85 \mathrm{a}$ & $20.07 \mathrm{a}$ & $140.38 \mathrm{a}$ \\
\hline RB92579 & $12.50 \mathrm{a}$ & 101.64 a & $12.86 \mathrm{~b}$ & $12.90 \mathrm{~b}$ & 82.57 a & $18.76 \mathrm{~b}$ & $129.96 \mathrm{~b}$ \\
\hline RB928064 & $8.74 \mathrm{~b}$ & $63.97 \mathrm{c}$ & $13.56 \mathrm{a}$ & $13.65 \mathrm{a}$ & $85.05 \mathrm{a}$ & $19.47 \mathrm{~b}$ & $135.94 \mathrm{a}$ \\
\hline RB93509 & $11.74 \mathrm{a}$ & 99.39 a & $13.16 \mathrm{~b}$ & $11.96 \mathrm{c}$ & $78.66 \mathrm{~b}$ & $18.29 \mathrm{~b}$ & $121.35 \mathrm{c}$ \\
\hline RB942520* & $10.21 \mathrm{~b}$ & $71.25 \mathrm{c}$ & $13.36 \mathrm{a}$ & $14.43 \mathrm{a}$ & $84.30 \mathrm{a}$ & $20.70 \mathrm{a}$ & $144.67 \mathrm{a}$ \\
\hline RB942849* & $9.21 \mathrm{~b}$ & $80.52 \mathrm{~b}$ & $12.87 \mathrm{~b}$ & $11.64 \mathrm{c}$ & $79.23 \mathrm{~b}$ & $17.51 \mathrm{~b}$ & $119.97 \mathrm{c}$ \\
\hline RB942898* & $10.15 \mathrm{~b}$ & $74.85 \mathrm{c}$ & $13.42 \mathrm{a}$ & $13.69 \mathrm{a}$ & $85.88 \mathrm{a}$ & $19.29 \mathrm{~b}$ & $136.21 \mathrm{a}$ \\
\hline RB942991* & $10.58 \mathrm{a}$ & $76.79 \mathrm{c}$ & $12.71 \mathrm{~b}$ & $13.77 \mathrm{a}$ & $84.44 \mathrm{a}$ & $19.48 \mathrm{~b}$ & $137.02 \mathrm{a}$ \\
\hline RB943066* & $9.80 \mathrm{~b}$ & $67.89 \mathrm{c}$ & $13.11 \mathrm{~b}$ & $14.35 \mathrm{a}$ & $83.83 \mathrm{a}$ & $20.63 \mathrm{a}$ & $143.50 \mathrm{a}$ \\
\hline RB943161* & $10.10 \mathrm{~b}$ & $72.43 \mathrm{c}$ & $14.05 \mathrm{a}$ & $13.98 \mathrm{a}$ & $85.70 \mathrm{a}$ & $19.97 \mathrm{a}$ & $139.86 \mathrm{a}$ \\
\hline RB943365 & $9.91 \mathrm{~b}$ & $69.79 \mathrm{c}$ & $12.75 \mathrm{~b}$ & $13.98 \mathrm{a}$ & $84.53 \mathrm{a}$ & $19.76 \mathrm{a}$ & $140.46 \mathrm{a}$ \\
\hline RB943538 & $8.23 \mathrm{~b}$ & $62.16 \mathrm{c}$ & $13.63 \mathrm{a}$ & $13.08 \mathrm{~b}$ & $83.55 \mathrm{a}$ & $18.99 \mathrm{~b}$ & $131.99 \mathrm{~b}$ \\
\hline RB952675* & $9.05 \mathrm{~b}$ & $66.54 \mathrm{c}$ & $13.52 \mathrm{a}$ & $13.65 \mathrm{a}$ & $85.32 \mathrm{a}$ & $19.40 \mathrm{~b}$ & $135.19 \mathrm{a}$ \\
\hline RB952900* & $8.80 \mathrm{~b}$ & $66.06 \mathrm{c}$ & $13.94 \mathrm{a}$ & $13.31 \mathrm{~b}$ & $83.90 \mathrm{a}$ & $19.39 \mathrm{~b}$ & $133.46 \mathrm{~b}$ \\
\hline RB953180* & $9.40 \mathrm{~b}$ & $68.72 \mathrm{c}$ & $12.83 \mathrm{~b}$ & $13.57 \mathrm{a}$ & $83.31 \mathrm{a}$ & $19.51 \mathrm{~b}$ & $135.84 \mathrm{a}$ \\
\hline RB953281* & $9.68 \mathrm{~b}$ & $73.04 \mathrm{c}$ & $13.72 \mathrm{a}$ & $13.23 \mathrm{~b}$ & $84.54 \mathrm{a}$ & $19.03 \mathrm{~b}$ & $131.98 \mathrm{~b}$ \\
\hline SP78-4764 & $10.11 \mathrm{~b}$ & $72.43 \mathrm{c}$ & $13.96 \mathrm{a}$ & $13.88 \mathrm{a}$ & $85.24 \mathrm{a}$ & $19.87 \mathrm{a}$ & $141.81 \mathrm{a}$ \\
\hline SP79-1011 & $11.27 \mathrm{a}$ & $79.47 \mathrm{~b}$ & $13.49 \mathrm{a}$ & $14.20 \mathrm{a}$ & $84.92 \mathrm{a}$ & $20.26 \mathrm{a}$ & $141.81 \mathrm{a}$ \\
\hline SP81-3250 & $11.66 \mathrm{a}$ & $82.83 \mathrm{~b}$ & $13.58 \mathrm{a}$ & $14.02 \mathrm{a}$ & $84.62 \mathrm{a}$ & $20.10 \mathrm{a}$ & $140.07 \mathrm{a}$ \\
\hline MÉDIA GERAL & 10,09 & 74,68 & 13,29 & 13,58 & 83,94 & 19,52 & 136,03 \\
\hline
\end{tabular}

Médias seguidas da mesma letra, nas colunas, pertencem ao mesmo grupo pelo teste de agrupamento de Scott e Knott, a $5 \%$ de probabilidade. * Clones promissores do PMGCA/UFRPE/RIDESA 
então que os ciclos de colheita são ambientes contrastantes, por causa dos fatores climáticos, e, de acordo com Rosse et al., (2002), exercem influência sobre os caracteres avaliados.

A fonte de variação relacionada com a interação (genótipo x cortes), para as variáveis TPH, TCH, PCC, PZA, BRIX e ATR, mostrou significância a $1 \%$ de probabilidade $(\mathrm{P}<0,01)$, e, para a variável FIB, a $5 \%$ de probabilidade $(\mathrm{P}<0,05)$. Pode-se constatar que a expressão desses importantes componentes de produção também depende em grande parte dos fatores climáticos, que são diferenciados nos ciclos de colheita de cana planta, soca e ressoca. Melo et al., (2009) encontraram resultados semelhantes para essas variáveis, com exceção de PZA, ao avaliar o desempenho agroindustrial de variedades de cana-de-açúcar no litoral sul de Pernambuco.

Observa-se nos parâmetros genéticos estimados, expostos na Tabela 4, que o componente de variância genética foi superior ao da variância da interação (genótipos $\mathrm{x}$ corte), para as variáveis TCH, FIB, PCC, PZA, BRIX e
ATR. Constata-se que a expressão dos caracteres avaliados nos diferentes ciclos de colheita, apesar das diferenças climáticas, é devida, em sua maior parte, aos componentes genéticos, com exceção da variável TPH, que é um indicativo de longevidade nas socarias.

Confirmando esse potencial de longevidade das socarias, os valores da herdabilidade média oscilaram de média a alta magnitude; entretanto, é possível obter ganhos genéticos significativos com a prática da seleção e maior ganho nos ciclos de colheita, sobretudo para a variável TCH, cujo coeficiente de variação genético $(\mathrm{CVg})$ alcançou valor acima de dez, indicando ser a variável que reteve a maior variabilidade genética (Oliveira et al., 2008).

Estão apresentados na Tabela 5 os valores médios para os caracteres toneladas de pol por hectare (TPH), toneladas de cana por hectare (TCH), fibra (FIB), pol \% corrigida (PCC), pureza (PZA), teor de sólidos solúveis (BRIX) e açúcar total recuperável (ATR), avaliados na $1^{\text {a }}$ época de colheita (início de safra).

Tabela 6. Valores econômicos, em reais por hectare, dos 11 clones e 15 variedades de cana-de-açúcar avaliados na fase de competição de variedades na $1^{a}$ época de colheita (início de safra), considerando os cultivos de cana planta, soca e ressoca (três cortes) em grupos de experimentos conduzidos na microrregião Centro de Pernambuco, Usina Petribú, Lagoa de Itaenga-PE, anos agrícolas 2005/2006, 2006/2007 e 2007/2008, Recife (PE), 2011

\begin{tabular}{|c|c|c|c|c|}
\hline Genótipos & TCH & ATR & R\$/Kg ATR** & $* \mathbf{R} \$ / \mathbf{h a}$ \\
\hline Q138 & $69,79 \mathrm{c}$ & $131,79 b$ & 0,50 & $4.598,81$ \\
\hline RB72454 & $76,79 \mathrm{c}$ & $135,77 \mathrm{a}$ & 0,50 & $5.212,89$ \\
\hline RB75126 & $82,1 b$ & $130,69 b$ & 0,50 & $5.364,82$ \\
\hline RB813804 & $62 c$ & $144,91 \mathrm{a}$ & 0,50 & $4.492,21$ \\
\hline RB863129 & $78,18 \mathrm{c}$ & $133,07 b$ & 0,50 & $5.201,71$ \\
\hline RB867515 & $84,64 b$ & $138,73 a$ & 0,50 & $5.871,05$ \\
\hline RB872552 & $66,41 \mathrm{c}$ & $140,27 \mathrm{a}$ & 0,50 & $4.657,67$ \\
\hline RB892700* & $72,06 \mathrm{c}$ & $140,38 \mathrm{a}$ & 0,50 & $5.057,89$ \\
\hline RB92579 & $101,64 a$ & $129,96 \mathrm{~b}$ & $\mathbf{0 , 5 0}$ & $6.604,57$ \\
\hline RB928064 & $63,97 \mathrm{c}$ & $135,94 \mathrm{a}$ & 0,50 & $4.348,04$ \\
\hline RB93509 & 99,39a & $121,35 \mathrm{c}$ & $\mathbf{0 , 5 0}$ & $6.030,49$ \\
\hline RB942520* & $71,25 \mathrm{c}$ & $144,67 \mathrm{a}$ & 0,50 & $5.153,87$ \\
\hline RB942849* & $80,52 b$ & $119,97 \mathrm{c}$ & 0,50 & $4.829,99$ \\
\hline RB942898* & $74,85 \mathrm{c}$ & $136,21 \mathrm{a}$ & 0,50 & $5.097,66$ \\
\hline RB942991* & $76,79 \mathrm{c}$ & $137,02 \mathrm{a}$ & 0,50 & $5.260,88$ \\
\hline RB943066* & $67,89 \mathrm{c}$ & $143,5 \mathrm{a}$ & 0,50 & $4.871,11$ \\
\hline RB943161* & $72,43 c$ & $139,86 a$ & 0,50 & $5.065,03$ \\
\hline RB943365 & $69,79 \mathrm{c}$ & $140,46 a$ & 0,50 & $4.901,35$ \\
\hline RB943538 & $62,16 \mathrm{c}$ & $131,99 b$ & 0,50 & $4.102,25$ \\
\hline RB952675* & $66,54 \mathrm{c}$ & $135,19 a$ & 0,50 & $4.497,77$ \\
\hline RB952900* & $66,06 \mathrm{c}$ & $133,46 b$ & 0,50 & $4.408,18$ \\
\hline RB953180* & $68,72 \mathrm{c}$ & $135,84 a$ & 0,50 & $4.667,46$ \\
\hline RB953281* & $73,04 \mathrm{c}$ & $131,98 b$ & 0,50 & $4.819,91$ \\
\hline SP78-4764 & $72,43 c$ & $141,81 \mathrm{a}$ & 0,50 & $5.135,65$ \\
\hline SP79-1011 & $79,47 b$ & $141,81 \mathrm{a}$ & 0,50 & $5.634,82$ \\
\hline SP81-3250 & $82,83 b$ & $140,07 \mathrm{a}$ & 0,50 & $5.801,00$ \\
\hline
\end{tabular}

* Clones promissores do PMGCA/UFRPE/RIDESA

** Preço médio * Valor bruto

Rev. Ceres, Viçosa, v. 59, n.5, p. 677-683, set/out, 2012 
Nota-se que, para as variáveis TPH, FIB e BRIX, ocorreu a formação de dois grandes grupos. Para a variável $\mathrm{TPH}$, a média geral foi de 10,09, variando entre 8,23 e 12,50, valores estes referentes ao clone RB943538 e à variedade RB92579, respectivamente. Apresentaram melhores desempenhos em TPH os genótipos enquadrados no grupo (a): RB92579, RB867515, RB93509, SP81-3250, SP79-1011, RB75126, RB942991 e RB72454. Silva (2008), analisando clones RB de cana-de-açúcar no litoral sul de Pernambuco, encontrou resultado semelhante para média da variável TPH, variando entre 8,12 e 12,92 , para as variedades RB863129 e RB763710, respectivamente.

Em relação à produtividade agrícola $(\mathrm{TCH})$, a magnitude dos valores variou de $62 \mathrm{t} / \mathrm{ha}$, para a variedade RB813804, a 101,64 t/ha, para a variedade RB92579, que, juntamente com a variedade RB93509, apresentou produtividade de 99,39t/ha; as três compuseram o grupo (a), comportando-se como as mais produtivas, para o início de safra, na microrregião em estudo. Em relação a variável PCC, destaca-se a variedade RB813804, com $14,48(\%)$, sendo superior a todos os genótipos avaliados. Apresentaram os melhores desempenhos para PCC os genótipos que compuseram o grupo (a): RB813804, RB942520, RB943066, SP79-1011, RB892700, RB872552, RB867515, SP81-3250, RB943161, RB943365, SP78-4764, RB942991, RB942898, RB72454, RB928064, RB952675 e RB953180.

A variedade RB813804 apresentou superioridade sobre os demais genótipos estudados em relação à variável ATR, com média de 144,91, acima da média geral observada, que foi de 136,03. Apresentaram melhores desempenhos em ATR os genótipos que fazem parte do grupo (a): RB813804, RB942520, RB943066, SP79-1011, SP78-4764e RB943365.

Com o objetivo de eleger os genótipos mais produtivos, por meio da análise da rentabilidade econômica bruta, apresentada na Tabela 6 , pode-se visualizar que, na média de três cortes, os produtores da microrregião canavieira da Zona Centro de Pernambuco têm a sua disposição, como os dois genótipos mais produtivos, para colheita no início da safra, as variedades, RB92579 e RB93509, que apresentaram renda bruta da ordem de R \$ 6.604 e $\mathrm{R} \$ 6.030$ reais por hectare, respectivamente.

\section{CONCLUSÕES}

As variedades e os clones avaliados apresentam potencial para fins de melhoramento, com destaque para o caráter TCH.

Para a microrregião Centro de Pernambuco, os genótipos RB92579 e RB93509 são os mais produtivos e de maior rentabilidade, sendo indicados para colheita no início da safra.

\section{REFERÊNCIAS}

Bastos IT, Barbosa MHP, Cruz CD, Burnquist WL, Bressiani JÁ \& Silva FL (2003) Análise dialélica em clones de cana-de-açúcar. Bragantia, 62:199-206.

Cruz CD (2006) Programa Genes: estatística experimental e matrizes. $1^{\mathrm{a}}$ ed. Viçosa, UFV. 285p.

Dutra Filho JA, Melo LJOT, Resende LV, Anunciação Filho CJ \& Bastos GQ (2011a) Aplicação de técnicas multivariadas no estudo da divergência genética em cana-de-açúcar. Revista Ciência Agronômica, 42:185-192.

Dutra Filho JÁ, Bastos GQ, Resende LV, Simões Neto De, Melo LJOT \& Daros E (2011b) Avaliação agroindustrial e dissimilaridade genética em progênies e variedades RB de canade-açúcar. Agropecuária Técnica, 32:55-61.

Espironelo A, Pommer CV, Pereira JCVA \& Igue T (1988) Avaliação de variedades IAC de cana-de-açúcar das séries de 1965 e 1966 e de outras cultivadas no estado de São Paulo. Bragantia, 47:83-92.

Fernandes A (2003) Cálculos na agroindústria da cana-de-açúcar. $2^{\mathrm{a}}$ ed. Piracicaba, EME. 240p.

Ferreira A, Barbosa MHP, Cruz CD, Hoffmann HP, Vieira MAS, Bassinello AI \& Silva MF (2005) Repetibilidade e número de colheitas para a seleção de clones de cana-de-açúcar. Pesquisa Agropecuária Brasileira, 40:761-767.

Gomes FP (1990) Curso de estatística experimental. 13 a ed. Piracicaba, USP. 467p.

Gonçalves GM, Viana AP, Bezerra Neto FV, Pereira MG \& Pereira TNS (2007) Seleção e herdabilidade na produção de ganhos genéticos em maracujá amarelo. Pesquisa Agropecuária Brasileira, 42:193-198.

Koffler NF, Lima JFWF, De Lacerda MF, Santana JF \& Silva MA (1986) Caracterização Edafo-climática das Regiões Canavieiras do Brasil: Pernambuco. $1^{\mathrm{a}}$ ed. Piracicaba, IAA/PLANALSUCAR. $78 \mathrm{p}$.

Mamede RQ, Bassinello AI, Casagrande AA \& Miocque JYJ (2002) Potencial produtivo de clones RB de cana-de-açúcar no Município de Nova Europa-SP. STAB-Açúcar, Álcool e Subprodutos, 20:32-35.

Melo LJOT, Oliveira FJ, Bastos GQ, Anunciação Filho CJ \& Reis OV (2006) Interação genótipo x ciclos de colheita de cana-deaçúcar da Zona da mata Norte de Pernambuco. Bragantia, 65:197-205.

Melo LJOT, Oliveira FJ, Bastos GQ, Anunciação Filho CJ \& Reis OV (2009) Desempenho agroindustrial de variedadees de canade-açúcar na zona da mata litoral sul de Pernambuco. Ciência e Agrotecnologia, 33:684-691.

Oliveira RA, Daros E, Bespalhok-Filho JC, Zambon JLC, Ido OT, Weber H, Resende MDV \& Zeni-Neto H (2008) Seleção de famílias de cana-de-açúcar via modelos mistos. Scientia Agrária, 9:269-274.

Rosse LN, Vencovsky R \& Ferreira DF (2002) Comparação de métodos de regressão para avaliar a estabilidade fenotípica em cana-de-açúcar. Pesquisa Agropecuária Brasileira, 37:25-32.

Scott AJ \& Knott M (1974) A cluster analysis method for grouping means in the analysis of variance. Biometrics, 30:507-512.

Silva GC (2008) Seleção de clones RB de cana-de-açúcar no litoral sul da zona da mata de Pernambuco utilizando técnicas multivariadas. Dissertação de Mestrado. Universidade Federal Rural de Pernambuco, Recife. 109p.

Stupiello JP (2002) Conversando com a cana. STAB - Açúcar, Álcool e Subprodutos, 20:38. 